
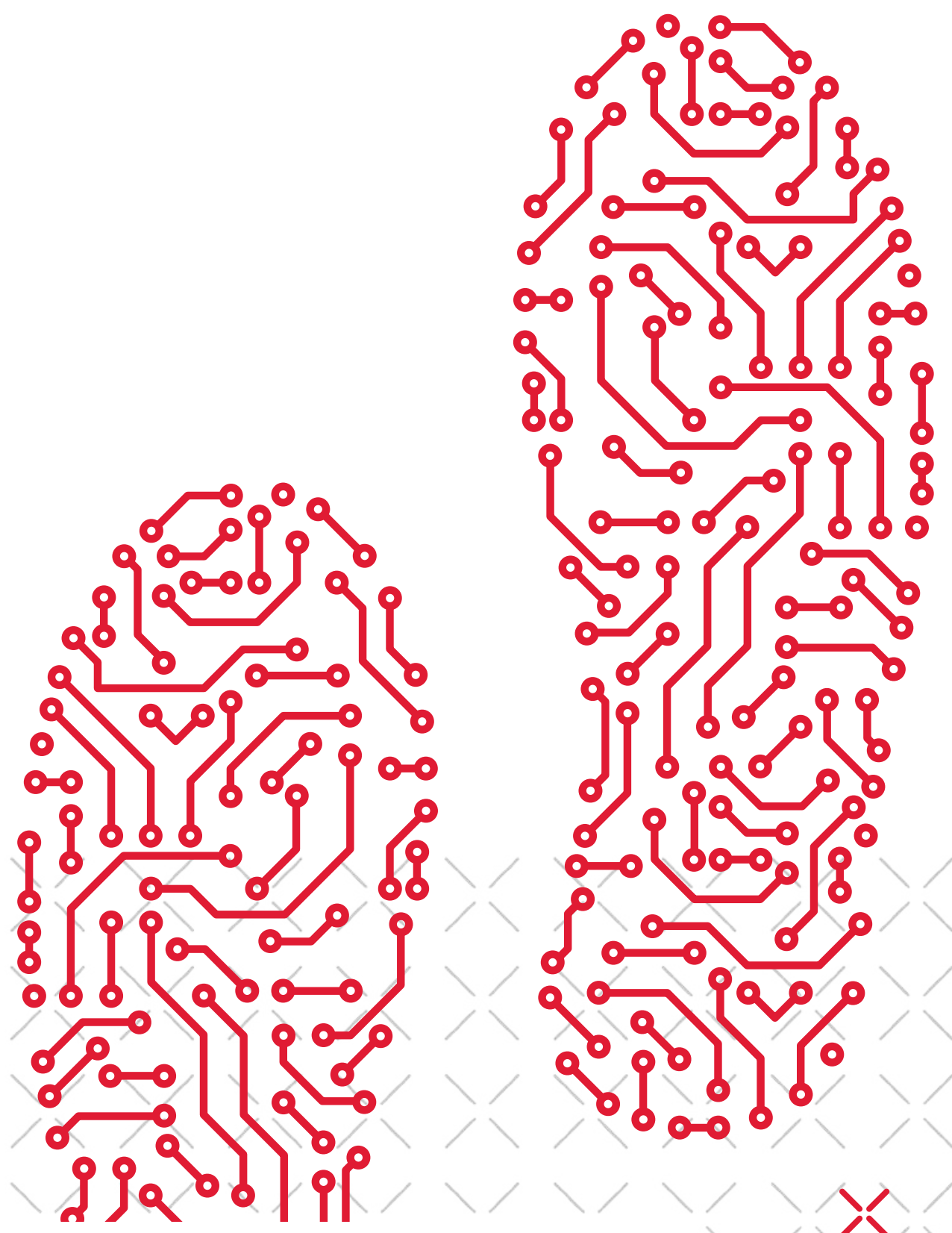

Digital footprints can be very accurate in the assessment of personality characteristics of large target groups and in designing more effective mass communication tools.

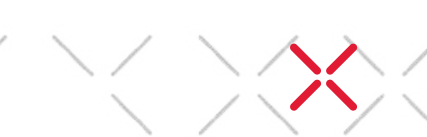




\title{
Using Consumers' Digital Footprints for More Persuasive Mass Communication
}

\author{
Sandra Matz and Michal Kosinski
}

KEYWORDS

Digital Footprints, Personality Assessment, Psychological Targeting, Persuasion,

Digital Mass Communication

THE AUTHORS

Sandra Matz

Professor of Management and Organizational Behavior, Columbia Business School, New York, NY, USA sm4409@gsb.columbia.edu

\section{Michal Kosinski}

Professor of Organizational Behavior, Stanford Graduate School of Business, Stanford, CA, USA michal@michalkosinski.com
What convinces one person might not convince another ׳ Persuasive mass communication is aimed at encouraging large groups of people to believe in and act on the communicator's viewpoint. It is used by governments to encourage healthy behaviors, by marketers to acquire and retain consumers, and by political parties to mobilize the voting population. Persuasive communication is particularly effective when tailored to people's unique psychological characteristics and motivations.

But how can marketers or other communicators obtain reliable psychological profiles of millions of users? How can they best navigate the landscape of psychological mass persuasion against the backdrop of data protection regulations such as the GDPR which restrict the storage and use of personal data? And what are the potential risks and pitfalls associated with hyper-personalized mass persuasion?

Digital footprints are remarkably predictive $\ltimes$ More and more human activities - such as social interactions, entertainment, shopping, and searching for information - happen, at least partially, in the digital space. Powered by better hardware and software, and fueled by the emergence of computational social science, these traces of human activity can be used to make highly personal inferences about their owner's preferences, habits and psychological characteristics.

Even relatively basic digital records of human behavior, such as Facebook likes, tweets or transaction records, can be used to automatically and accurately estimate a wide range of personal attributes including political ideology, sexual orientation 


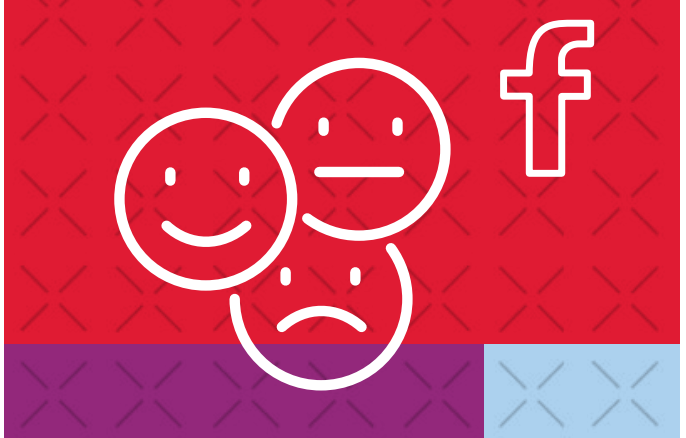

BOX 1

\section{How Likes can reveal your personality}

\section{Show me what you Like and I can tell you who you are}

One of our studies focused on Facebook Likes to predict a range of personal attributes. Based on a large sample with 170 Likes per participant on average, we used regression analysis to predict the attributes listed in Figure 1. Prediction accuracy ranged from $95 \%$ for ethnic origin and $93 \%$ for gender to $60 \%$ for inferring whether users' parents stayed together or were separated before users were 21 (see Figure 1). In a follow-up study, we showed that computer-based predictions of people's personality traits from Facebook Likes were more accurate than the judgements made by co-workers, friends and family members, and almost as accurate as those made by a person's spouse.

FIGURE 1 > How Likes reveal your personality

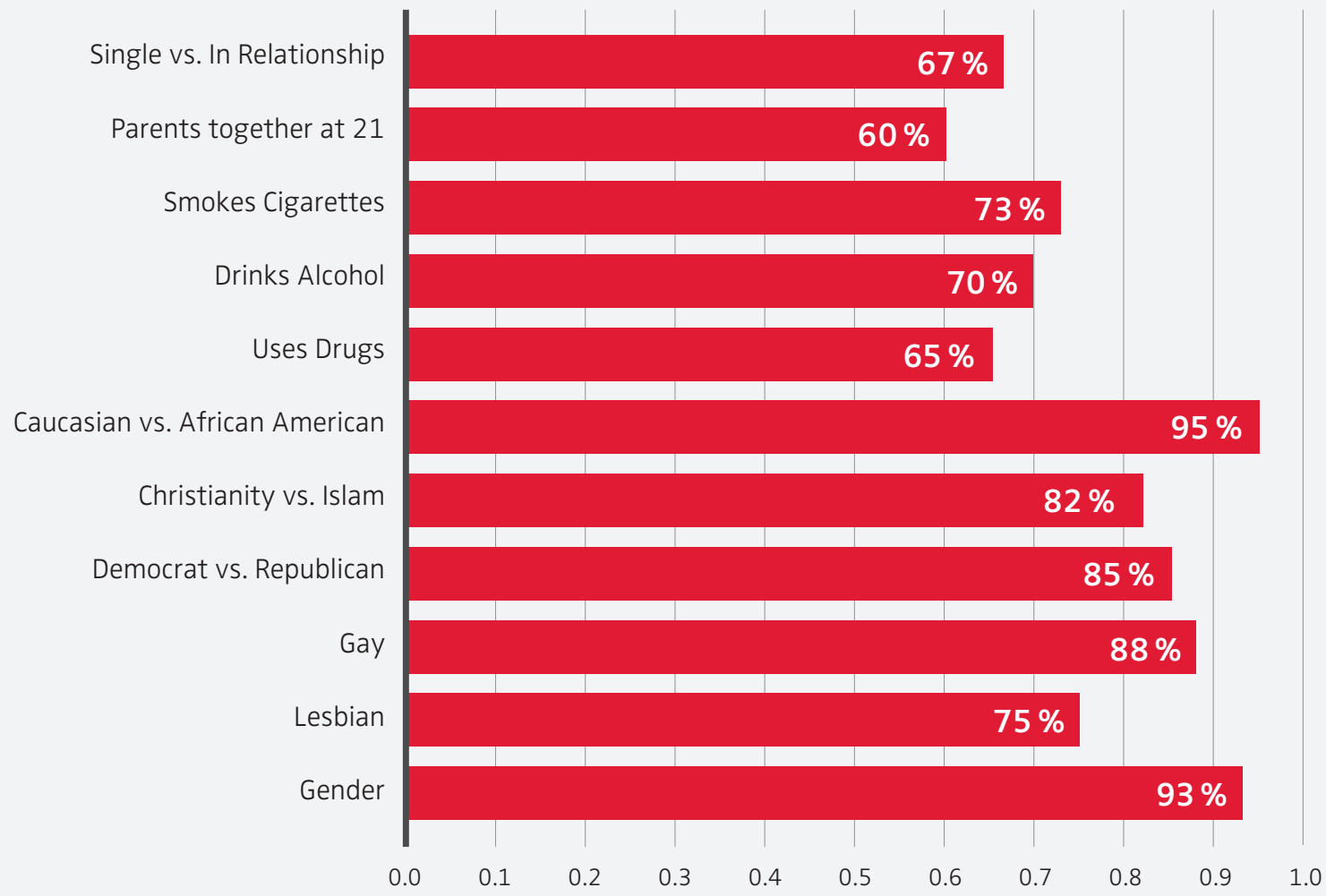

(from Kosinski, Stillwell and Graepel, 2013) 
BOX 2

How language reveals your personality

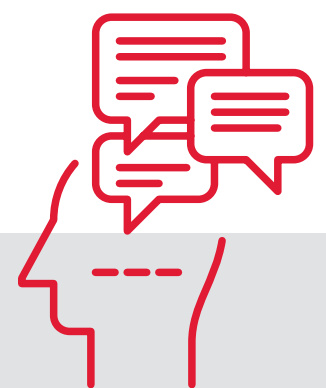

Show me your posts and I will know how you feel

In another study, we used the language of Facebook status messages to assess the personality of the poster. We included words, phrases, emoticons, punctuation (such as "!!!"), and web-language expressions (such as "omg" or "wtf") and counted their occurrences within a voluntary sample of over 66,000 participants who wrote at least 1,000 words (with an average of 4.1 words per post) during the time span of the study.

Based on word frequencies and topics used, we built a language model to predict personality traits. The language analyses showed high correlations with self-reported measures of the five commonly used personality traits: openness, conscientiousness, extraversion, agreeableness, and neuroticism.

Vast amounts of Likes and textual posts are publicly available on social media networks and represent a massive source of rich psychological data. While psychological assessments were primarily questionnaire-based and difficult to scale, predictive models based on Likes, posts, and tweets can be administered easily to millions of people to accurately capture their psychological characteristics. This ability, in turn, opens the door for attempts to persuade/ influence large groups of people more effectively in the form of psychological targeting.

and personality - attributes that most people would typically assume to be private. Automated assessments on the basis of digital footprints may not only be more accurate and less prone to cheating and misrepresentation than traditional, scale-based personality assessments, but they can also permit measurement over time to detect temporal trends and intra-individual changes in behavior. In a series of studies, we have tested and demonstrated how digital footprints can indeed be very accurate in the assessment of personality characteristics (Box 1 and Figure 1) of large target groups and in designing more effective mass communication tools (Figure 2).

Better results for ads tailored to recipients' personality In three Facebook campaigns that reached over 3.5 million individuals, we used personality traits predicted from Facebook Likes to test the effectiveness of psychologically-tailored advertising. We found that matching the content of persuasive appeals to individuals' personality significantly altered their behavior as measured by clicks and purchases. Persuasive appeals that were matched to people's extraversion or openness-to-experience level resulted in up to $40 \%$ more clicks and up to $50 \%$ more purchases than their mismatching counterparts or counterparts that were not personalized.

For example, in one of these studies, we tailored the persuasive advertising messages for a UK-based beauty retailer to recipients' extraversion - a personality trait reflecting the extent to which people seek and enjoy company, excitement, and stimulation. Figure 2 shows how we segmented and targeted the ad audience based on their Facebook Likes, and how the campaign translated into conversions. We selected 10 Likes characterized by the highest and lowest aggregate extraversion scores respectively, and targeted users with 5 introverted and 5 extroverted messages. Averaged across the campaigns, users in the matching conditions were over $50 \%$ more likely to purchase from the online store than users in the mismatching conditions.

The bright and dark sides of persuasive mass communication $\times$ Our findings suggest that the application of psychological targeting makes it possible to influence the behavior of large groups of people by tailoring persuasive appeals to the psychological needs of the target audiences. On the one hand, this method holds potential benefits for helping individuals make better decisions and lead healthier and happier lives. On the other hand, we see a number of potential pitfalls related to manipulation, data protection and privacy violations. While both opportunities and challenges are bountiful, here we only focus on three important ones.

$>$ Better products, services and consumer decisions $\times$ Predicting users' individual attributes and preferences can be used to improve numerous products and services. For 
FIGURE $2>$ How addressing consumers tailored to Likes-based personality assessment translates into higher conversion rates

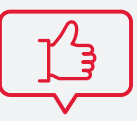

ลิกิกิ้ก

10 target Likes most indicative of high extraversion

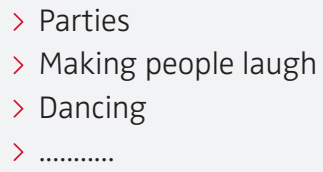

Exposure to ad targeted to high extraversion

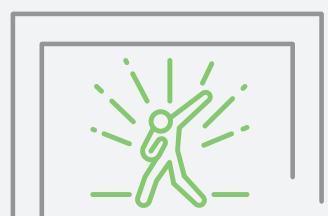

Dance like no one's watching (but they totally are)

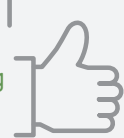

Audience segmentation using the psychological trait "extraversion"

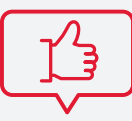

คิ้นก้น

10 target Likes most indicative of low extraversion

$>$ Sanctuary

$>$ Stargate

$>$ Computers

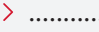

\section{Exposure}

to ad targeted to

low extraversion

\section{Advertising \\ Campaign \\ on Facebook}

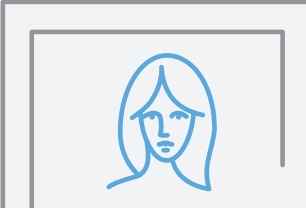

Beauty doesn't have to shout

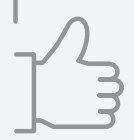

Introverted Ads

Extroverted Ads

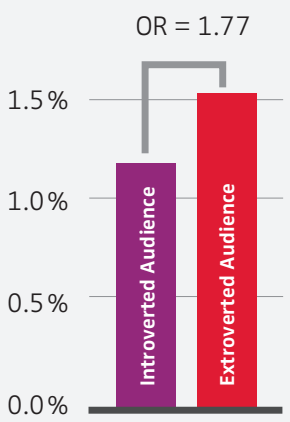

Averaged across the campaigns, users in the matching conditions were $\mathbf{1 . 5 4}$ times more likely to purchase from the online store than users in the unmatching conditions.
$\mathrm{OR}=1.40$

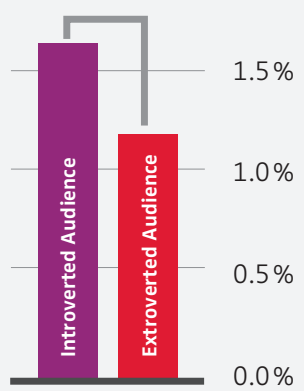

Conversion Rates 
instance, digital systems and devices such as online stores and car displays could be designed to adjust their functions to best fit each user's inferred profile. By doing so they can help individuals overcome the challenge of "choice overload" and reduce unwanted spam, and assist them in finding the products and services they are most interested in.

The relevance of marketing and product recommendations could be improved by adding psychological dimensions to current user models. For example, online insurance advertisements might emphasize security when facing emotionally unstable users - a trait that can be predicted fairly well - but stress potential threats when dealing with emotionally stable ones. If the presented information is more relevant, it will be easier for consumers to make choices that suit their needs and make them happy, as well as make decisions that seem difficult in the short-term but benefit them in the long-run (e.g. saving or exercising more).

$>$ Unwanted disclosure of private matters $`$ The predictions of personal attributes from digital traces without the individual's awareness and consent may have considerable negative consequences. Commercial companies, government institutions, or even one's Facebook friends could use software to infer attributes such as intelligence, sexual orientation, or political views that an individual may not have intended to share. In the wrong hands, such predictions could pose a threat to an individual's well-being, freedom, or even life. For example, psychological targeting could be abused to covertly exploit weaknesses in people's characters and persuade them to take action against their own best interest or engage in immoral or unlawful dealings.

But even when intentions are benign and there is no direct intent for manipulation and data abuse, unintended consequences can arise and harm consumers. For example, a major U.S. retail network used customer shopping records to predict pregnancies of its female customers and send them well-timed and well-targeted offers. While in some contexts an unexpected flood of vouchers for prenatal vitamins and maternity clothing might be welcome, it could also be annoying or even tragic in other situations. In the worst-case scenario, revealing her pregnancy to her partner, parents, or other family members could threaten a woman's well-being or even safety.

\section{Data privacy protection is challenging beyond the GDPR}

׳ To date, legislative approaches in the U.S. and Europe have focused on increasing the transparency of how information is gathered and ensuring that consumers have a mechanism to "opt out" of tracking. The GDPR effectuated by the EU in 2018 has considerably restricted the ability to store and use consumers' personal data, giving more power and control to the individual.

However, the psychological targeting procedure using Facebook Likes or language as outlined in this article challenges the extent to which existing and proposed legislation can protect individual privacy. Personal inferences can be made even without having direct access to individuals' data. Although we used indirect group-level targeting in a way that was anonymous at the individual level and thus preserved - rather than invaded - participants' privacy, the same approach could also be used to reveal individuals' intimate traits without their awareness by following consumers who click on an ad or make a purchase.

None of the legislative measures currently in place or in discussion fully address the techniques described here: Our empirical experiments were performed without collecting or processing any individual-level information. Yet leveraging group-level insights through the Facebook advertising platform made it possible to influence the behavior of thousands of people on the basis of their psychological traits. Consequently, even today's most progressive data protection regulations might not adequately address the potential abuse of online information in the context of psychological targeting, highlighting the need for further policy interventions and regulations.

$\downarrow$

\section{FURTHER READING}

Kosinski, M.; Stillwell, D. J. and Graepel, T. (2013):

"Private Traits and Attributes are Predictable from Digital Records of Human Behavior", Proceedings of the National Academy of Sciences, Vol. 110 (15), 5802-5805.

https://doi.org/10.1073/pnas.1218772110

Matz, S. C.; Kosinski, M.; Nave, G. and Stillwell, D. J. (2017): "Psychological Targeting as an Effective Approach to Digital Mass Persuasion." Proceedings of the National Academy of Sciences, 114 (48), 12714-12719. https://doi.org/10.1073/pnas.1710966114

Park, G.; Schwartz, A. H.; Eichstaedt, J. C.; Kern, M. L.; Kosinski, M.; Stillwell, D. J.; Ungar, I. H. and Seligman, M. E. P. (2015): “Automatic personality assessment through social media language." Journal of Personality and Social Psychology, Vol. 108 (6), 934-952. http://dx.doi.org/10.1037/pspp0000020 